\title{
PREFERÊNCIA DE OVIPOSIÇÃO E SUA RELAÇÃO COM O DESEMPENHO DE DIAPHANIA HYALINATA (L., 1758) (LEPIDOPTERA: CRAMBIDAE) EM CUCURBITÁCEAS
}

\author{
C.A. Guedes ${ }^{1}$, V.F. Silva ${ }^{2}$, G.S. Cruz ${ }^{2}$, A.P. Lôbo ${ }^{1}$, A.A.C. Teixeira' ${ }^{2}$, V. Wanderley-Teixeira ${ }^{2}$ \\ ${ }^{1}$ Universidade Federal Rural de Pernambuco, Departamento de Agronomia, Rua Dom Manoel de Medeiros, \\ s/nº, CEP 52171-900, Recife, PE, Brasil. E-mail: valeria@dmfa.ufrpe.br
}

RESUMO

Diaphania hyalinata L. (Lepidoptera, Pyralidae) destaca-se como principal praga da família Cucurbitaceae. Este lepidóptero é oligófago de hábito polífago, que desperta largo interesse de estudos da sua ecologia comportamental por motivos de manejo em culturas de grande importância econômica como o meloeiro (Cucumis melo L.). Dada esta situação, trabalhos básicos de entendimento de aspectos da ecologia são necessários para a melhoria das formas de manejo, principalmente no aspecto do monitoramento populacional. Diante disso, a presente pesquisa teve como objetivo verificar se a escolha do hospedeiro exercida por fêmeas de $D$. hyalinata corresponde ao melhor desempenho da prole e como os hospedeiros influenciam sua dinâmica populacional. Testes de oviposição com chance de escolha foram realizados em melão, melancia, maxixe, bucha, melãode-são-caetano e pepino, para estabelecimento de um ranking de preferência. Para o desempenho das lagartas e reprodução foi escolhido o melão (dentre o mais preferido) e a bucha e o pepino (dentre os hospedeiros menos preferidos). Os resultados suportam a hipótese de que a escolha de hospedeiro é relacionada com o desempenho da prole e que D. hyalinata demonstrou possuir plasticidade ao lidar com diferentes hospedeiros o que pode ser um indicativo de capacidade de adaptação a recursos alimentares que flutuam em termos de qualidade e disponibilidade. No entanto, de modo geral, esta plasticidade tem custos elevados, principalmente em termos reprodutivos.

PALAVRAS-CHAVE: Broca-das-cucurbitáceas, preferência alimentar, planta hospedeira, desempenho da prole, relação planta-inseto.

\begin{abstract}
OVIPOSITION PREFERENCE AND ITS RELATION TO THE PERFORMANCE OF DIAPHANIA HYALINATA (L., 1758) (LEPIDOPTERA: CRAMBIDAE) IN CUCURBITS. Diaphania hyalinata L. (Lepidoptera, Pyralidae) stands out as a major pest of the Cucurbitaceae family. This insect is oligophagous with a polyphagous habit, which arouses wide interest in studies of their behavioral ecology for reasons of pest management in crops of high economic importance such as muskmelon (Cucumis melo L.). Given this situation, basic studies aimed at an understanding of ecological aspects of this pest are needed to improve pest management systems, especially in the aspect of population monitoring. Given this, the present study was aimed to determine whether the choice of the host exerted by females of $D$. hyalinata corresponds to the best performance of the offspring and how the hosts influence their population dynamics. Oviposition-choice tests were performed on melon, watermelon, cucumber, loofah, balsam apple and cucumber, to establish a ranking of preference. For the performance of larvae and reproduction the melon was chosen (among the most preferred hosts) along with loofah and cucumber (among the least preferred hosts). The results support the hypothesis that the choice of host is related to the performance of the offspring and D. hyalinata has demonstrated plasticity in dealing with different hosts which may be indicative of ability to adapt to fluctuating food resources in terms of quality and availability. However, generally this plasticity is expensive, especially in reproductive terms.
\end{abstract}

KEY WORDS: Pickleworm, feeding preferences, host plants, offspring performance, plant insect relationship.

${ }^{2}$ Universidade Federal Rural de Pernambuco, Departamento de Morfologia e Fisiologia Animal, Recife, PE, Brasil. 


\section{INTRODUÇÃO}

A relação planta-inseto acontece desde o período carbonífero, a cerca de 300 milhões de anos, sendo considerada a maior fonte geradora de diversidade dos organismos terrestres (GulLaN; Cranston, 2007). Nessa interação, quando uma planta modifica o seu sistema de defesa químico ou físico, existe a formação de um provável nicho vago, possibilitando ao seu detentor receber os benefícios da isenção de predadores, o que provavelmentelhe permitirá acumular mais energia para a sua manutenção e reprodução, com melhor possibilidade de competir em sua comunidade, tendo chance de preponderar no ambiente. Por outro lado, os insetos que ocupam a mesma comunidade têm a probabilidade de serem selecionados a utilizarem esse novo organismo como recurso alimentar e, caso sejam capazes de quebrar a defesa, terão a possibilidade de ocupar um novo nicho, podendo tornar-se especialista na utilização deste recurso, o que pode gerar uma nova espécie, com o passar dos anos (Gullan; Cranston, 2007).

As defesas químicas das plantas contra herbívoros desempenham papel chave no processo de co-evolução e são constituídos de várias substâncias como os compostos fenólicos (taninos, benzenoides etc.), terpenoides (monoterpenoides, sesquiterpenoides etc.), alcaloides, glicosídeos cianogênicos e glicosinolatos, entre outros (HARBORNE, 1999; BRUCE; WoodCock, 2005). Podem provocar efeitos diversos nos insetos como deterrência alimentar ou de oviposição, redução da taxa de alimentaçãoe capacidade de metabolização do alimento, além de depressão da sua função imune (LilL; MARQUis, 2001; HAVIOla et al., 2007). As defesas proporcionadas pelos compostos secundários restringem o rol de hospedeiros associado a uma determinada planta e geram a necessidade de classificação especial da relação planta-inseto, quanto à capacidade de utilização dos hospedeiros como recurso alimentar (GULLAN; CRANSTON, 2007).

Geralmente a fase imatura dos insetos tem aptidão limitada para a locomoção, sendo o encontro do hospedeiro e distribuição da prole de responsabilidade dos adultos (JANZ, 2002). Dessa forma, a capacidade de avaliação da planta hospedeira torna-se fator primordial para sobrevivência dos neonatos, principalmente dos insetos monófagos e oligófagos (BERNAYs; CHAPMAN, 1994; NyLin; GOTTHARD, 1998). A compreensão dos fatores determinantes da relação entre escolha do hospedeiro e preferência alimentar (que envolve a escolha para oviposição) é uma importante chave para o entendimento de muitos aspectos da ecologia dos insetos (PRICE et al., 1998). A relação entre a escolha e desempenho da prole influencia a dinâmica popula- cional dos herbívoros por estar estreitamente ligado à história de vida desses organismos (NyLIN, 2001). Acredita-se que a escolha exercida pelas fêmeas seja relacionada ao melhor desempenho da prole (JANZ, 2002), mas alguns trabalhos demonstram resultados discordantes, promovendo a necessidade de novos estudos para elucidar as questões do processo de seleção do hospedeiro (AWMACK; LEATHER 2002; JALLOW; ZALUCKI, 2003).

Na família Cucurbitaceae, que compreende cerca de 118 gêneros e 825 espécies predominantemente tropical, existem algumas plantas cultivadas para a produção de alimentos, fibras e fitoterápicos (LENZIet al., 2005) que têm como principal herbívoro Diaphania hyalinata L. (Lepidoptera, Pyralidae) (PRATISSOLI et al., 2007). Este lepidóptero é oligófago de hábito polífago, que desperta largo interesse de estudos da sua ecologia comportamental por motivos de manejo em culturas de grande importância econômica como o meloeiro (Cucumis melo L.). Dada esta situação, trabalhos básicos de entendimento de aspectos da ecologia são necessários para a melhoria das formas de manejo, principalmente no aspecto do monitoramento populacional. Assim, a pesquisa objetivou: I - investigar se a escolha por fêmeas de $D$. hyalinata quanto ao hospedeiro para oviposição tem relação direta com o desempenho da prole, sob condições de laboratório, II - se a hierarquia de escolha do hospedeiro para oviposição tem correlação com o desempenho da prole, sendo que as fêmeas escolhem plantas que contribuam diretamente para a sua aptidão darwiniana, III - lagartas criadas em hospedeiro preferencial têm rápido desenvolvimento larval, maior fecundidade, fertilidade e longevidade, além da qualidade de ovos.

\section{MATERIAL E MÉTODOS}

\section{Criação de D. hyalinata}

A pesquisa foi realizada a partir da criação mantida no Laboratório de Comportamento de Insetos, Área de Fitossanidade da UniversidadeFederal Rural de Pernambuco-UFRPE, à temperatura de $26 \pm 2^{\circ} \mathrm{C}$, umidade relativa de $70 \pm 10 \%$ e fotofase de 12 horas.

Folhas de abobrinha-caserta (Cucurbita pepo L.) (Top Seed), cultivadas em casa-de-vegetação, foram destacadase seus pecíolosimersos emágua em garrafas plásticas de $500 \mathrm{~mL}$ para manutenção da turgidez e oferecidas aos adultos de $D$. hyalinata para oviposição em gaiolas de madeira e organza com $80 \times 60 \times 60 \mathrm{~cm}$. Após o período deincubação, as lagartas de D. hyalinata recémeclodidas forammantidasnasmesmascondições até a fase de pupa. Após emergências, os adultos foram transferidos para as gaiolas, ondeera oferecida solução de sacarose a $10 \%$ como fonte de alimento. 
Escolha de hospedeiros para oviposição de $D$. hyalinata com chance de escolha

Gaiolas de madeira e organza de $1 \times 1 \times 1 \mathrm{~m}$ foram utilizadas para essa etapa, sendo distribuídas garrafas plásticas de $500 \mathrm{~mL}$ contendo $200 \mathrm{~mL}$ de água e diferentes folhas de cucurbitáceas, a saber: melão (C. melo), melão-de-são-caetano (Momordica charantia L.), pepino (Cucumis sativus L.), maxixe (Cucumis anguria L), melancia [Citrullus lanatus (Thunb.) Matsum. \& Nakai] e bucha (Luffa spp.). O experimento constou de seis tratamentos (hospedeiros) em 16 repetições utilizando dez fêmeas previamente acasaladas em cada repetição. As garrafas foram distribuídas de forma equidistantes em um arranjo hexagonal, alternando as posições das folhas dos hospedeiros nas diferentes gaiolas. Após 24 horas o número total de ovos em cada hospedeiro foi contabilizado e o índice de preferência de oviposição foi estabelecido por meio do cálculo de porcentagem de ovos encontrados.

Os dados foram submetidos à análise de variância e teste de média de Duncan a 5\% de significância. Antes da realização da análise, foi realizada uma análise exploratória dos dados, verificando-se a homogeneidade de variâncias e normalidade pelos testes de Bartllete Cochran e Lilliefors pelo programa estatístico SAEG.

Após analise estatística selecionou-se um dentre os melhores hospedeiros e dois hospedeiros menos preferidos para os próximos testes de impacto sobre a biologia, desempenho das lagartas e efeito sobre a reprodução de D. hyalinata.

Impacto dos hospedeiros selecionados sobre a biologia de $D$. hyalinata

Em tubos de ensaio de $1 \times 5 \mathrm{~cm}$ contendo $1 \mathrm{~mL}$ de ágar a 1\% (para manter a turgidez da folha), após resfriamento e solidificação, foram colocados ovos com cerca de $48 \mathrm{~h}$ sobre pedaços de folha de $1 \mathrm{~cm}^{2}$ de cada hospedeiro como alimento para lagartas recém-eclodidas de $D$. hyalinata, e acompanhados até o início da fase larval. Nessa etapa foi observada a mortalidade das lagartas nas primeiras $72 \mathrm{~h}$. Posteriormente, as lagartas foram transferidas para recipientes maiores para os próximos ensaios.

Efeito dos hospedeiros selecionados no desempenho de lagartas de $D$. hyalinata

Após três dias da eclosão as lagartas foram transferidas para garrafas plásticas de $200 \mathrm{~mL}$, contendo esponja umedecida em água; folíolo dos respectivos hospedeiros para alimentação delas; e coberto com organza para permitir aeração.

Foi acompanhado o tempo de desenvolvimento, considerando a data de eclosão até a formação da pré-pupa de lagartas que se transformaram em machos e fêmeas do inseto. Também foi acompanhada a taxa de ganho de massa corpórea diária durante o desenvolvimento larval. Para isso, cerca de 40 lagartas foram pesadas diariamente, sendo relacionada a taxa de ganho de massa corpórea ao sexo, além do tipo de hospedeiro. Utilizou-se um delineamento de medidas repetidas para evitar pseudoreplicações com o acompanhamento diário da taxa de ganho de massa corpórea (VON ENDE, 1993). A verificação do sexo foi feita no segundo dia do período pupal, através da observação do último segmento do abdômen do inseto.

Efeito dos hospedeiros selecionados na reprodução de D. hyalinata

Foi acompanhada a emergência de adultos de $D$. hyalinata nos diferentes hospedeiros, sendo, posteriormente, formados casais entreespécimes originários de mesmos hospedeiros. Ao final dos acasalamentos a longevidade e fecundidade foram determinadas através da observação diária do número de ovos ao longo da vida das fêmeas.

\section{RESULTADOS}

Verificou-se que D. hyalinata tem um o comportamento de oviposição diferenciado quanto às espécies de hospedeiros testados, sendo a melancia e o meloeiro aqueles que receberam semelhantemente maior número de ovos, enquanto que as plantas de maxixe, bucha, melão-de-são-caetano e pepino apresentaram, equivalentemente, menores quantidades de ovos ( $F=4,26, G L=89 ; P=0,00162)$ (Fig. 1). A partir desses resultados foram selecionados o melão como melhor hospedeiro e bucha e pepino como os hospedeiros menos preferidos.

A taxa de ganho de massa corpórea das lagartas de D. hyalinata diferenciou quanto ao sexo, tempo e a espécie de hospedeiro utilizado (Fig. 2). A taxa de ganho de massa corpórea foi de um modo geral, crescente para os hospedeiros melão, bucha e pepino ao longo do período de desenvolvimento, sendo máxima ao oitavo dia. A taxa de ganho de massa corpórea foi semelhante entre os sexos das lagartas para os diferentes hospedeiros testados, excluindo para as lagartas que se alimentaram em folhas de pepino, onde as fêmeas apresentaram menor ganho da taxa de massa corpórea. Lagartas macho e fêmea que se alimentaram de folhas de bucha apresentaram um ganho superior de massa corpórea no quinto dia do tempo de desenvolvimento, mas voltaram a ter incrementos seme-lhantes às outras lagartas que se alimentaram de folhas de melão e pepino nos dias consecutivos. 


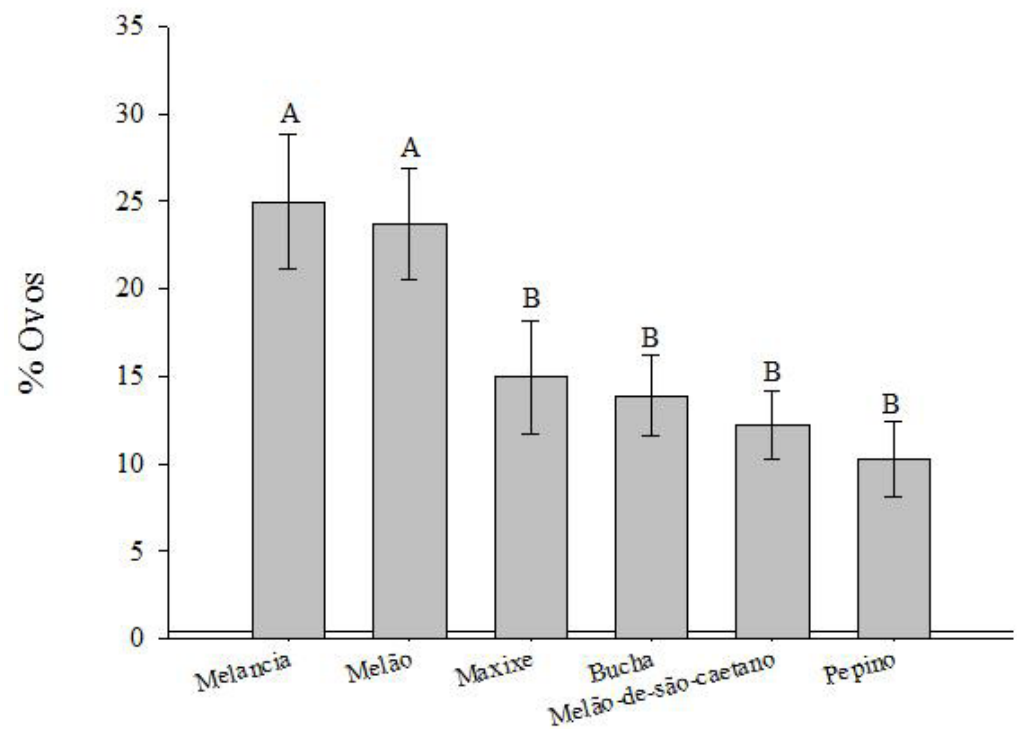

Hospedeiros
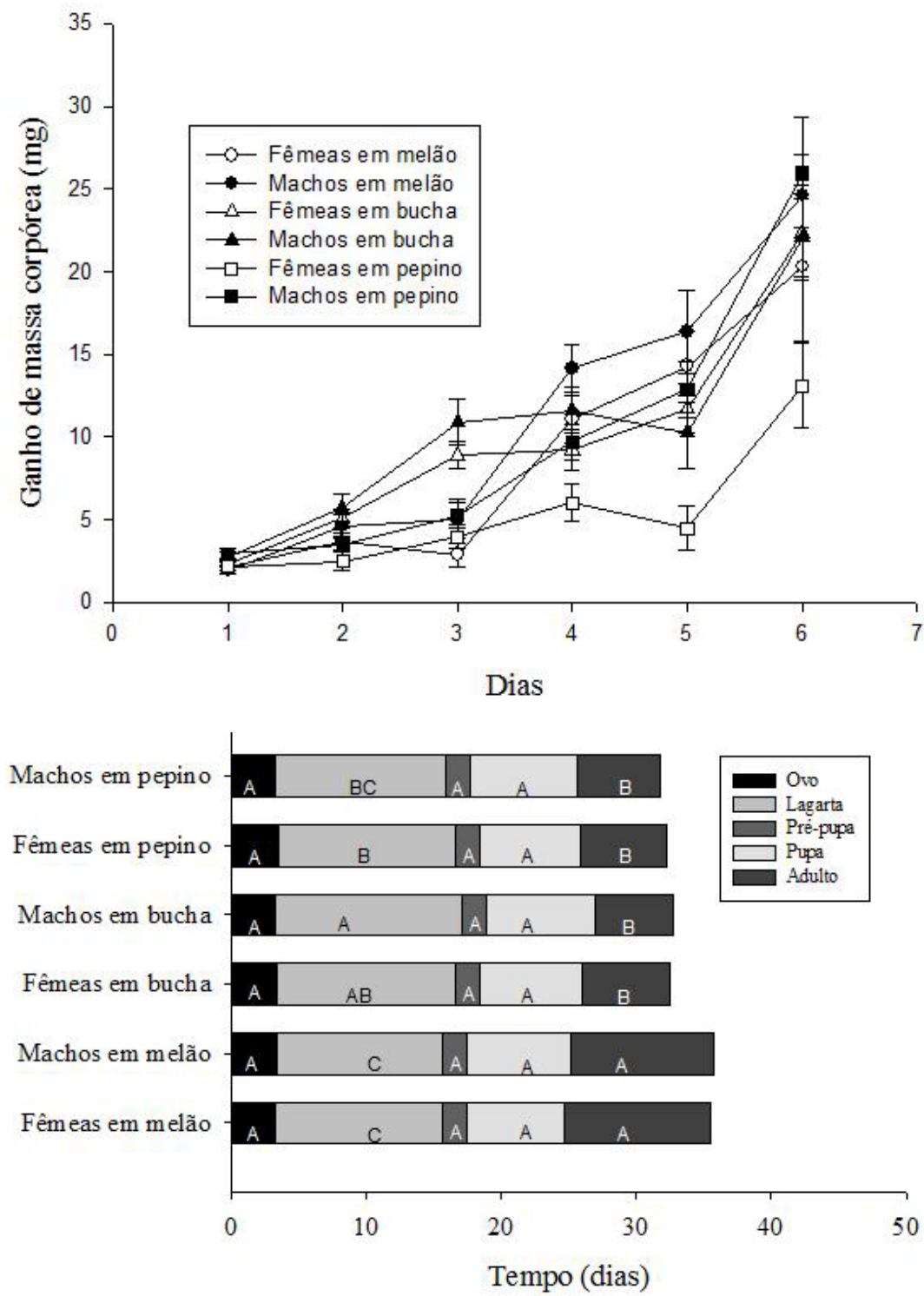

Fig. 1 - Preferência de oviposição de Diaphania hyalinata entre diferentes cucurbitáceas. Médias seguidas de mesma letra não diferem entre si pelo teste de Ducan a 5\% de significância.

Fig. 2 - Efeito de diferentes cucurbitáceas no ganho de peso de lagartas machos e fêmeas de Diaphaniahyalinata. Os círculos, quadrados e triângulos representam a média e barras verticais representam o erro-padão. Teste de Ducan a 5\% de significância.
Fig. 3 - Influência de meloeiro, bucha e pepino nos tempos dos estádios de desenvolvimento de Diaphania hyalinata. Médias seguidas de mesma letra entre barras de mesma cor não diferem entre si pelo teste de Ducan a 5\% de significância. 


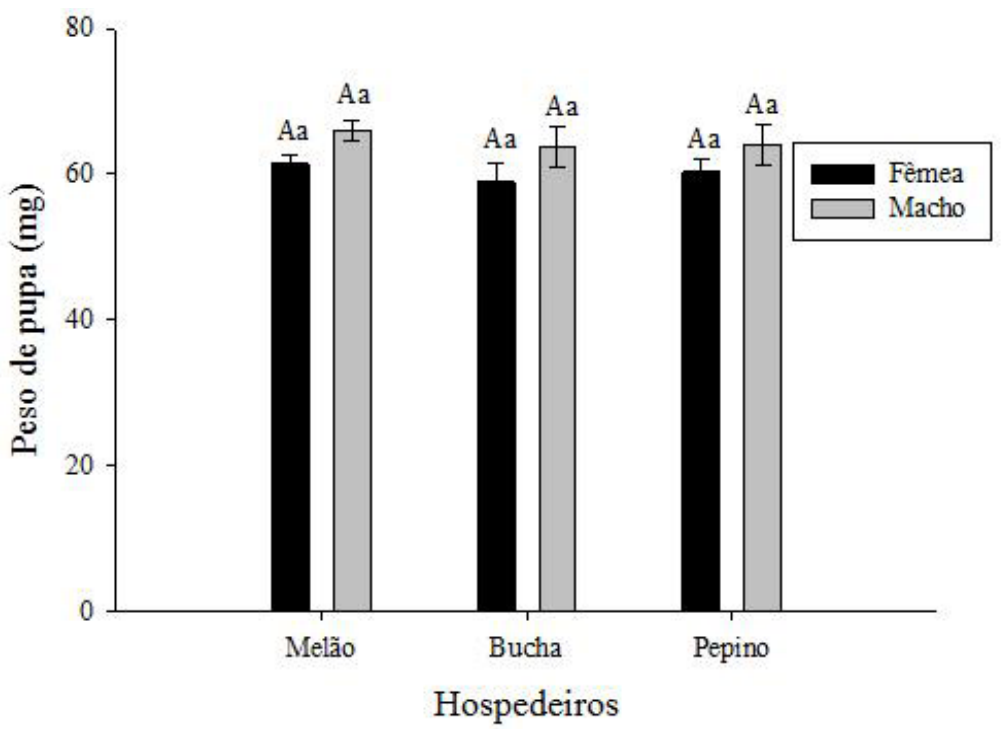

Fig. 4 - Efeito de três espécies de cucurbitáceas no peso de pupas de machos e fêmeas de Diaphania hyalinata. Médias seguidas de mesma letra maiúscula entre sexo e letra minúscula entre espécies de hospedeiros não diferem entre si pelo teste de Ducan a 5\% de significância.

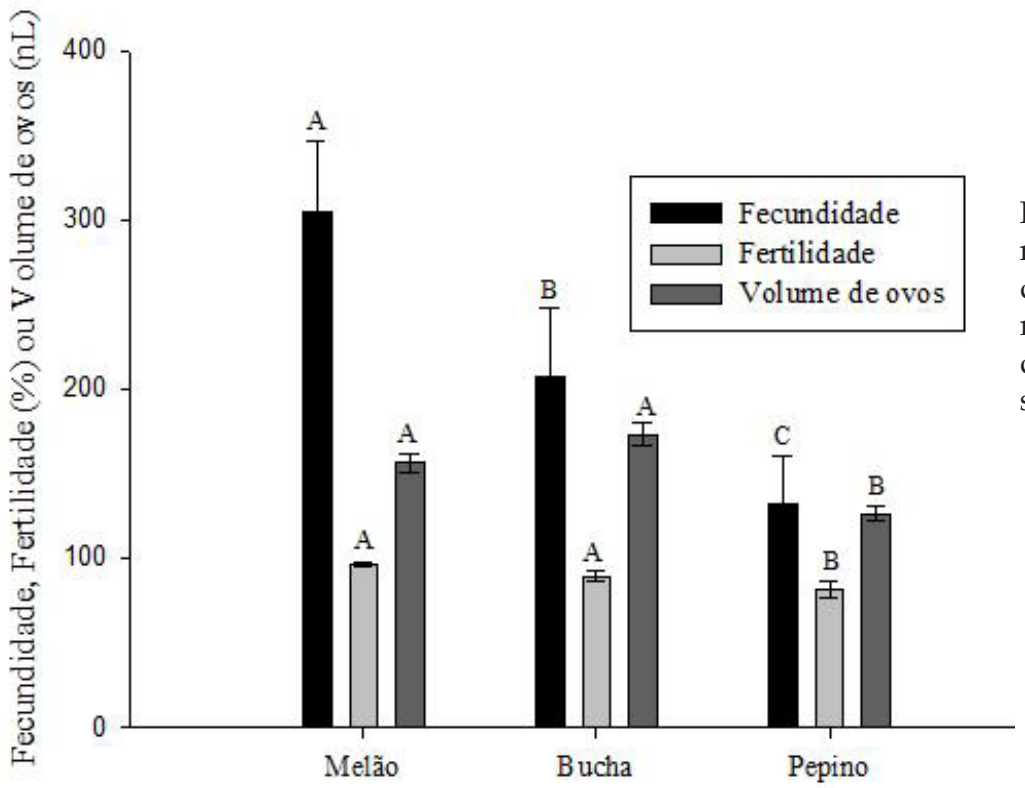

Hosnedeirns

Otempodafaselarval diferiuquantoaosexoehospedeiros utilizados (Fig. 3). As lagartas macho efêmea que sealimentaram defolhas demeloeirodesenvolveram-se mais rápido do que nos outros hospedeiros e de forma semelhante, também foi equivalente aos machos que se alimentaram defolhas depepino. Otempodafaselarval para machos e fêmeas de lagartas que se alimentaram defolhas de pepino e bucha foi semelhante, excetuando para machos que se alimentou de folhas de bucha. Estes apresentaram o tempo de desenvolvimento maior do que machos e fêmeas que se alimentaram de pepino $(\mathrm{F}=4,45, \mathrm{GL}=112 ; \mathrm{P}=0,001)$.

O tempo de pré-pupa foi semelhante entre os sexos para todos os hospedeiros utilizados como fonte de alimento $(\mathrm{F}=0,052, \mathrm{GL}=110 ; \mathrm{P}>0,05)$. De forma semelhante, a fase de pupa também não apresentou diferença significativa no tempo de desenvolvimento para os sexosdosinsetos criadosnos trêshospedeiros $(\mathrm{F}$ $=2,54, G L=62 ; P>0,05)$ (Fig. 3). Não houve diferenças significativas no peso de pupas entre os sexos de $D$. hyalinataeentrepupasdediferenteshospedeiros(Fig.4).

O tempo da fase adulta de machos e fêmeas das lagartas que se alimentaram de folhas de meloeiro foi superior ao tempo dos adultos das lagartas que se alimentaram de folhas de bucha e pepino $(\mathrm{F}=3,93$, $\mathrm{GL}=61 ; \mathrm{P}=0,00375$ (Fig. 3).

A emergência dos adultos foi equivalente entre os espécimes dos três hospedeiros, onde para os insetos que se alimentaram de pepino apresentou $80,23 \%$, seguido da bucha que foi de $82,35 \%$, e por último o melão, apresentando $78,79 \%$. Não houve diferenças significativas para o tempo de incubação 
dos ovos das lagartas que se alimentaram dos três hospedeiros ( $F=0,874, G L=113 ; P>0,05)$ (Fig. 3). Porém, houve diferença na viabilidade dos ovos, apresentado pelas lagartas que se alimentaram de meloeiro $91,36 \%$ de viabilidade do que as lagartas que se alimentaram de pepino $(78,22 \%)$ e a bucha $(75 \%)$, além da fecundidade e volume de ovos (Fig. 5).

\section{DISCUSSÃO}

As fêmeas mostraram preferência para hospedeiros específicos de cucurbitáceas sendo a oviposição concordante com o melhor desempenho da prole. Em insetos herbívoros, existem diversos exemplos de correlação positiva entre preferência da fêmea e subsequente desempenho da sua prole. Por exemplo, fêmeas do díptero Euura lasiolepis E.L. Smith, 1968 (Hymenoptera: Symphyta) preferem brotos quecrescem rapidamente para a oviposiçãono qual a sobrevivência larval é alta (CRAIG et al., 1989). Entretanto, NYLIN; JANZ (1993) relataram que fêmeas de Polygonia c-album L. (Lepidoptera: Nymphalidae) ovipositam nos hospedeiros que asseguram elevada taxa deeclosão para larvas e sugerem que a qualidade de hospedeiro é o fator mais importante da seleção dessa espécie.

Outrosparâmetrosdemedidadaaptidãodarwiniana (fitness) como sobrevivência ou massa pupal não diferiu entre hospedeiros. Estes resultados sugerem que melão é superior nutricionalmente para o crescimento de D. hyalinata. Entretanto, no começo do período larval as lagartas que se alimentaram em folhas de bucha cresceram mais rápido no terceiro dia, mas voltaram a ter o ritmo de crescimento igual às outras lagartas que se alimentaram dos outros hospedeiro.

A taxa de crescimento rápido é importante para herbívoros multivoltino como $D$. hyalinata em um país de clima tropical, pois assegura maior oportunidade para colonização de plantios ricos em recursos nutricionais como são os plantios de meloeiro no Nordeste brasileiro. Este fato também é importante pela redução da exposição aos inimigos naturais como predadores e parasitóides comuns aos plantios. Provavelmente, as fêmeas de D. hyalinata foram moldadas pelo processo evolutivo a utilizarem hospedeiros que favoreçam o crescimento rápido e também com alta taxa de reprodutiva. Desta forma, os resultados deste estudo suportam a teoria de que as fêmeas escolhem seus hospedeiros baseados no desempenho ótimo da prole.

JANZ (2002) sumariou as razões de muitos estudos terem falhado em encontrar correlação positiva entre escolha e desempenho da prole. Isto inclui, por exemplo, a seleção de hábitat ótimo próximo ao hospedeiro favorável ao desenvolvimento da prole ou a inabilidade das fêmeas identificarem o melhor hospedeiro para a prole. Este estudo encontrou diferença significante entre fêmeas em selecionar locais para oviposição indicando que poderia haver diferenças genéticas em uso do hospedeiro e seleção.

Compostos secundários podem estar envolvidos no limitado desempenho das lagartas de $D$. hyalinata em bucha e principalmente em plantas do pepino. As fêmeas tiveram baixo ganho de massa corpórea principalmente nos últimos três dias da fase larval, o que provavelmente contribui para a reduzida fertilidade, fecundidade e tamanho dos ovos. Alguns compostossecundárioscomo, por exemplos, os taninos são reconhecidamente redutores da digestibilidade do alimento (PANDA; KHUSH, 1995) e estes podem ser a explicação para o desempenho delarvas fêmeas em plantas de pepino. Por outro lado, os machos que se alimentaram tanto de bucha quanto de pepino não tiveram este fraco desempenholarval provavelmente devido a menor necessidade de acúmulo de energia voltada para a reprodução, visto que os custos energéticos neste gênero para formar gametas ser menor. Mesmo assim os machos que se alimentaram de bucha e pepino tiveram menor tempo de vida adulta, o que em termos reprodutivo pode comprometer a competividade reprodutiva.

Os resultados encontrados no presente estudo demonstram que as fêmeas de $D$. hyalinata preferem plantas que favoreçam ao desempenho da prole. Como a maioria dos experimentos de laboratórios utilizados para acessar a relação preferência e desempenho (THOMPSON, 1988; JALLOW; ZALUCKI, 2003) o presente trabalho não avaliou alguns aspectos ecológicos que são importantes no desempenho larval e sobrevivência em campo como a abundância do hospedeiro, parte do hospedeiro e sua arquitetura, a presença de inimigos naturais e microclima. Foram restringidas as partes das plantas utilizadas às folhas mais jovens, não utilizando folhas mais velhas e senescentes. Isto pode ter proporcionado grandes diferenças em termos nutricionais, principalmente em termos defolhas seccionadas. Consequentemente, o desempenho larval pode ser diferente do que é encontrado na natureza. Diferentes partes das plantas são reconhecidas em proporcionar diferentes desempenhos para os insetos (AWMACK; LEATHER, 2002). Certos componentes da aptidão darwiniana como, por exemplo, poder de atração das fêmeas, capacidade competitiva dos machos por acasalamentos, capacidade das fêmeas em evitar sítios de oviposição com inimigos naturais presentes podem ter sido subestimados ou difícies de avaliar em laboratório. Portanto, os dados do presente estudo devem ser complementados por similar análise em campo ou em casa-de-vegetação, levando em consideração os fatores ecológicos que podem ser importantes 
para desempenho da prole. Em primeira análise, os resultados deste trabalho trazem o primeiro contato para o entendimento do processo de escolha do hospedeiro por D. hyalinata.

\section{CONCLUSÕES}

D. hyalinata demonstrou possuir plasticidade ao lidar com diferentes hospedeiros pertencentes à família Cucurbitaceae o que pode ser um indicativo de capacidade de adaptação a recursos alimentares que flutuam em termos de qualidade e disponibilidade. No entanto, de modo geral, esta plasticidade tem custos elevados, principalmente em termos reprodutivos.

\section{AGRADECIMENTOS}

AoDepartamento de Agronomia da Universidade Federal Rural de Pernambuco, pela oportunidade de realização deste trabalho.

\section{REFERÊNCIAS}

AWMACK, C.S.; LEATHER, S.R. Host plant quality and fecundity in herbivorous insects Annual Review of Entomology, v.47, p.817-844, 2002.

BERNAYS, E.A.; CHAPMAN, R.F. Host-Plant Selection by Phytophagous Insects. 2.ed. London: Chapman \& Hall, 1994. 312p.

BRUCE, T.J.A.; WOODCOCK, C.W. Insect host location: a volatile situation. Trends in Plant Science, v.10, p.269274, 2005.

CRAIG, T.P.; ITAMI, J.K.; PRICE, P.W. A strong relationship between oviposition preference and larval performance in a shoot-galling sawfly. Ecology, v.70, n.6, p. 1691-1699, 1989.

GULLAN, P.J.; CRANSTON. P.S. Os insetos: um resumo de entomologia. São Paulo: Roca, 2007. 456p.

HARBORNE, J.B. Recent advances in chemical ecology. Natural Product Reports, v.16. p.509-523, 1999.

HAVIOLA, S.; KAPARI, L.; OSSIPOV, V.; RANTALA, M.J.; RUUHOLA, T.; HAUKIOJA, E. Foliar phenolics are differently associated with Epirrita autumnata growth and immunocompetence. Journal of Chemical Ecology, v.33, n.5, p.1013-1023, 2007.
JALLOW, M.F.A.; ZALUCKI, M.P. Relationship between oviposition preference and offspring performance in Australia Helicoperva armigera (Hübner) (Lepidoptera: Noctuidae). Australian Journal of Entomology, v.42, n.4, p.343-348, 2003.

JANZ, N. Evolutionary ecology of oviposition strategies. In: HILKER, M.; MEINERS, T. (Org.). Chemoecology of insect eggs and egg deposition. Berlin - Vienna: Blackwell Publishing, 2002. p.349-376.

LENZI, M.; ORTH, A.I.; GUERRA, T.M. Ecologia da polinização de Momordica charantia L. (Cucurbitaceae), em Florianópolis, SC, Brasil. Revista Brasileira de Botânica, v.28, n.3, p.505-313, 2005.

LILL, J.; MARQUIS, T. The effects of leaf quality on herbivore performance and attack from natural enemies. Ecology, v.126, p.418-428, 2001.

NYLIN, S. Life history perspectives on pest insects: What's the use? Austral Ecology, v.26, p.507-517, 2001.

NYLIN, S.; GOTTHARD, K. Plasticity in life history traits. Annual Review Entomology, v.43, p.63-83, 1998.

NYLIN, S.; JANZ, N. Oviposition preference and larval performance in Polygonia calbum (Lepidoptera: Nymphalidae): the choice between bad and worse. Ecology Entomology, v.18, p.394-398, 1993.

PANDA, N.; KHUSH, G.S. Host plant resistance to insects. Wallingford: CAB International, 1995, 431p.

PRATISSOLI, D.; POLANCZYK, R.A.; HOLTZ, A.M.; COCHETO, J.G.; TAMANHONI, T.; MILANEZ, A.M. Desenvolvimento da broca-das-cucurbitáceas em diferentes tipos de substratos alimentares. Horticultura Brasileira, v.25, p.98-601, 2007.

PRICE, P.W.; FERNANDES, G.W.; LARA, A.C.F.; BRAWN, J. Global patterns in local number of insect galling species. Journal of Biogeography, v.25, p.581-591, 1998.

THOMPSON, J.N. Evoluvionary ecology of the relationship between oviposition preference and performance of offspring in phytophagous insects. Entomologia Experimentalis et Applicata, v.47, p.3-14, 1988.

VON ENDE, C.N. Repeated-measures analyses: growth and other time-dependent measures. In: SCHEINER, S.; GUREVITCH, J. (Org.). Design and Analysis of Ecological Experiments. New York: Chapman \& Hall, 1993. p.13-137.

Recebido em 3/9/09

Aceito em 3/11/10 